\title{
Comportamento de cristalização de lipídios estruturados obtidos a partir de gordura do leite e óleo de girassol
}

\author{
Crystallization behavior of structured lipids by chemical interesterification of milkfat and sunflower oil
}

\author{
Juliana Neves RODRIGUES-RACT ${ }^{1}$, Lucia Nazareth COTTING ${ }^{1}$, \\ Tatyane Pereira POLTRONIERI ${ }^{1}$, Roberta Claro da SILVA ${ }^{1}$, Luiz Antonio GIOIELLI ${ }^{1 *}$
}

\begin{abstract}
Resumo
A finalidade do estudo foi avaliar o comportamento de cristalização de misturas binárias de gordura do leite e óleo de girassol em diversas proporções, bem como de seus correspondentes lipídios estruturados obtidos por interesterificação química. Foram usados conteúdo de gordura sólida, consistência, ponto de amolecimento e microscopia sob luz polarizada para avaliar as misturas antes e após a interesterificação. A adição de óleo de girassol e a interesterificação modificaram a cristalização da gordura do leite, pelos efeitos de diluição e rearranjo dos triacilgliceróis. Arquivos de vídeo em formato WMV foram compilados para estudar a cristalização a $20{ }^{\circ} \mathrm{C}$. Os vídeos tornaram possível a observação da estrutura cristalina formada e representaram uma ferramenta de grande importância para a pesquisa de cristalização de óleos e gorduras.
\end{abstract}

Palavras-chave: cristalização; lipídios estruturados; interesterificação química.

\begin{abstract}
The aim of this study was to evaluate the crystallization behavior of binary mixtures of milkfat and sunflower oil in different ratios and their respective structured lipids obtained by chemical interesterification. Solid fat content, consistency, softening point and polarized light microscopy were used to analyze the mixtures before and after interesterification. The addition of sunflower oil and the interesterification changed the milkfat crystallization, through the effect of the dilution and rearrangement of the triacylglycerols. Video files in WMV format were made in order to study the crystallization at $20^{\circ} \mathrm{C}$. The videos made it possible to observe crystal structure formation and they represent a tool of great importance in the fats and oils crystallization research.

Keywords: crystallization; structured lipids; chemical interesterification.
\end{abstract}

\section{Introdução}

O comportamento de cristalização de lipídios tem implicações muito importantes, principalmente no processamento industrial de produtos cujas características físicas dependem em grande parte de cristais de gorduras, como chocolates e margarinas, e separação de gorduras específicas a partir de gorduras naturais através do fracionamento (SATO, 2001; SILVA; ESCOBEDO; GIOIELLI, 2008; DÍAZ GAMBOA; GIOIELLI, 2006). A velocidade de formação dos cristais, o crescimento e as transformações polimórficas são importantes para se determinar o processo e as condições de armazenamento de óleos e gorduras (HERRERA et al., 1998).

Para o completo entendimento da reologia de gorduras plásticas, faz-se necessária a caracterização de sua microestrutura, já que gorduras plásticas consistem de uma rede cristalina em uma matriz oleosa contínua (GHOTRA; DYAL; NARINE, 2002; ROUSSEAU; HILL; MARANGONI, 1996a). Muitos fatores influenciam a cristalização dos lipídios, especialmente a maneira como estes são resfriados a partir de seu estado líquido, pois quando um óleo é resfriado, uma fase sólida se separa, cuja composição e quantidade dependem principalmente da taxa de resfriamento e das temperaturas inicial e final (ROUSSEAU; HILL; MARANGONI, 1996a; TIMMS, 1995).
Marangoni e Rousseau (1998) relacionaram uma série de fatores que influenciam as propriedades das redes cristalinas de gorduras plásticas, entre eles (i) tendência à cristalização, que inclui polimorfismo, tamanho, número, forma e comportamento de agregação dos cristais; (ii) composição química; (iii) proporção sólido/líquido; (iv) intersolubilidade entre os triacilgliceróis; (v) procedimento de cristalização, que inclui taxa de resfriamento, grau de super-resfriamento, tratamento mecânico e temperagem; e (vi) a presença de componentes como fosfolipídios e esteróis.

Como a velocidade de nucleação aumenta de forma exponencial com o aumento da supersaturação, enquanto a velocidade de crescimento é linearmente proporcional à supersaturação, o número de cristais aumenta e o tamanho do cristal diminui quando a cristalização ocorre a baixas temperaturas. $\mathrm{O}$ resfriamento rápido a baixa temperatura, seguido de agitação intensa leva à formação de cristais pequenos, como os encontrados na margarina. Por outro lado, o resfriamento lento sob agitação suave leva à formação de cristais grandes facilmente visíveis a olho nu. Com agitação 
suave, os cristais podem formar aglomerados de esferulitos com tamanhos de centenas de micrômetros (TIMMS, 1995; WRIGHT et al., 2001).

A complexidade e a flexibilidade dos triacilgliceróis permitem diferentes empacotamentos do mesmo conjunto de moléculas, levando à existência de diferentes formas polimórficas. Como a composição em triacilgliceróis da rede cristalina influencia diretamente o polimorfismo desta rede, seu comportamento de fusão está diretamente relacionado à sua composição, porém não existe nenhum método preditivo que relacione a composição em triacilgliceróis com o perfil de fusão de uma gordura (NARINE; MARANGONI, 1999b). A cristalização de gorduras é complicada não somente pelo polimorfismo, mas também porque ela usualmente ocorre a partir de fase líquida de multicomponentes, ao invés de um solvente puro, o que é mais comum em outros processos industriais de cristalização (HIMAWAN; STAROV; STAPLEY, 2006).

O comportamento reológico de gorduras plásticas é consequência das interações entre os cristais de gordura que se encontram em uma matriz tridimensional de gordura sólido-líquida agregada. As principais forças que levam à formação dessa rede são as forças atrativas de Van der Waals. A porção líquida da gordura, a fase contínua dispersa entre a rede de gordura agregada, em conjunto com a fração sólida, são responsáveis pelo comportamento viscoelástico. Assim, a quantidade de gordura cristalizada e os tipos de cristal presentes nessa matriz gordurosa têm importância primordial no comportamento reológico da gordura (ROUSSEAU; HILL; MARANGONI, 1996b; TANG; MARANGONI, 2006).

Os cristais são classificados em três principais formas $-\alpha, \beta$ ' e $\beta$ - de acordo com a estrutura da subcélula. A forma polimórfica $\beta$ 'é a mais comum na gordura do leite, com forma de esferulito. A maioria das gorduras apresenta polimorfismo monotrópico, isto é, quando ocorre uma transição (por exemplo, de $\beta$ ' para $\beta$ ), o empacotamento das cadeias moleculares do triacilglicerol se torna mais compacto, resultando em maior ponto de fusão (NARINE; MARANGONI, 1999b; ROUSSEAU et al., 1996).

O processo de cristalização é dividido em duas fases: nucleação e crescimento dos cristais. A nucleação envolve a formação de agregados de moléculas que excederam um tamanho crítico e são, portanto, estáveis. O processo de nucleação depende da supersaturação e do super-resfriamento (HERRERA et al., 1998; TIMMS, 1995).

Produtos com alto teor lipídico, como manteiga, margarinas, cremes vegetais e similares, devem apresentar proporção adequada entre as frações sólida e líquida para que o produto tenha a textura e a funcionalidade (isto é, espalhabilidade) desejada pelos consumidores (TORO-VASQUEZ et al., 2000). As propriedades reológicas macroscópicas das redes cristalinas lipídicas destes produtos são também extremamente importantes. Muitos dos atributos sensoriais, como espalhabilidade, sensação na boca e textura são dependentes da resistência mecânica da rede cristalina (PISKA et al., 2006).

Pesquisas na área de cristalização de gorduras utilizam metodologias de DSC (Differential Scanning Calorimetry ou
Calorimetria Diferencial de Varredura), difração de raios X, RMN (Ressonância Magnética Nuclear) e diferentes técnicas de microscopia (MARANGONI, 2005).

Apesar de terem sido publicados inúmeros estudos sobre a cristalização da gordura do leite envolvendo dados sobre dimensão fractal, número e tamanho dos cristais, reologia e cinética de cristalização, entre outras propriedades (ROUSSEAU; HILL; MARANGONI, 1996a; HERRERA; GATTI; HARTEL, 1999; METIN; HARTEL, 1998; WRIGHT et al., 2000; CAMPOS; NARINE; MARANGONI, 2002; BATTE; MARANGONI, 2005; LOPEZ et al., 2005), existem poucos dados quantitativos da cinética de formação e crescimento dos cristais (GRALL; HARTEL, 1992). Liang et al. (2006) relataram que é muito difícil descrever quantitativamente as propriedades da rede cristalina em função da complexidade e natureza randômica da estrutura de sistemas reais. Estes autores compararam diversos métodos de quantificação da microestrutura e introduziram o conceito de densidade da microestrutura, definido como o número de unidades microestruturais por unidade de volume do sistema. Os resultados mostraram que os parâmetros quantitativos de microestrutura, com exceção da dimensão fractal, puderam identificar as diferenças e similaridades das características microestruturais no sistema modelo lipídico estudado.

A velocidade de resfriamento de gorduras geralmente representa um fator de grande importância no tamanho, forma, número e forma polimórfica de cristais. Lopez et al. (2005) conduziram o resfriamento de gordura do leite de 1 a $3{ }^{\circ} \mathrm{C} /$ minuto e puderam correlacionar a velocidade de resfriamento das amostras com rearranjos estruturais das formas $\alpha, \beta^{\prime}$ e $\beta$. O resfriamento a $3{ }^{\circ} \mathrm{C} /$ minuto, por exemplo, por ser considerado rápido, levou à formação de diferentes cristais que ainda não estão em equilíbrio, ou seja, sofrerão transição polimórfica em algum momento.

Mazzanti et al. (2004) analisaram a gordura do leite por difração de raios X e observaram que o resfriamento da gordura do leite a altas velocidades e baixas temperaturas finais de cristalização resultou na formação de cristais na forma a metaestável, que posteriormente resultaram na formação de uma mistura de cristais dos tipos $\beta^{\prime}$ e $\beta$. Ao contrário, a cristalização realizada a baixas velocidades de resfriamento e altas temperaturas finais de cristalização resultou na formação de cristais apenas do tipo $\beta$ ', sem a presença de cristais dos tipos a ou $\beta$.

Alguns trabalhos recentes foram publicados apresentando, em suas versões on-line disponíveis na Internet, vídeos referentes à cristalização de óleos e gorduras (BATTE; MARANGONI, 2005; DIBILDOX-ALVARADO et al., 2004; LITWINENKO; SINGH; MARANGONI, 2004). Esses trabalhos, porém, não apresentaram uma análise quantitativa consistente das sequências de imagens.

O trabalho teve por objetivos: a) realizar misturas em diversas proporções de gordura do leite e óleo de girassol; b) efetuar interesterificação química entre os componentes das misturas; c) analisar o comportamento de cristalização das misturas antes e após a interesterificação por meio da microscopia sob luz polarizada. 


\section{Material e métodos}

\subsection{Material}

A gordura do leite foi obtida a partir de manteiga tipo extra sem sal (Paulista, Brasil), adquirida no varejo, por fusão completa à temperatura de $60-70{ }^{\circ} \mathrm{C}$ em forno de microondas para provocar quebra da emulsão e separação das fases gordurosa e aquosa. A fase aquosa contendo grande parte dos sólidos do leite foi descartada. A fase gordurosa, que apresentava turbidez devido à presença de sólidos do leite e de gotículas de fase aquosa, foi filtrada em papel de filtro em estufa a $50{ }^{\circ} \mathrm{C}$. Obteve-se, por fim, a gordura do leite pura que, quando fundida, apresentava cor amarela intensa e transparente e odor característico. Foi preparado um único lote de gordura do leite, que foi utilizado posteriormente na preparação de todas as misturas. A manteiga foi utilizada como fonte de gordura do leite neste trabalho pela simplicidade do processo de separação da gordura e também por representar a fonte mais rica em gordura entre todos os produtos lácteos disponíveis no mercado. $\mathrm{O}$ óleo de girassol refinado Liza (Cargill, Brasil) foi adquirido no varejo e utilizado sem tratamento adicional.

Para o estudo das interações em misturas ternárias de gorduras foi realizado um planejamento de 5 experimentos, apresentado na Tabela 1.

A gordura do leite é representada por $\mathrm{x}_{1}$ e o óleo de girassol é representado por $\mathrm{x}_{2}$, sendo que $\mathrm{x}_{1}+\mathrm{x}_{2}=1$ ou $100 \%$.

As misturas preparadas continham de $60 \%$ a $100 \%$ de gordura do leite, pois, para a preparação de um spread, bases gordurosas que contêm grandes quantidades de óleo apresentam pouca plasticidade e não têm aplicação. Os limites do componente gordura do leite e óleo de girassol foram estabelecidos com base nos resultados obtidos por Rodrigues (2002), Rodrigues, Anton e Gioielli (2003), Rodrigues, Gioelli e Anton (2003), Rodrigues e Gioielli (2003) e Rodrigues et al. (2004). As misturas foram preparadas nas proporções mencionadas, após fusão completa à temperatura de $70{ }^{\circ} \mathrm{C}$ e novamente solidificadas, sendo armazenadas sob resfriamento.

\subsection{Métodos}

\section{Interesterificação química}

Foram interesterificados $350 \mathrm{~g}$ de cada mistura, previamente seca em balão de vidro, sob pressão reduzida, acoplado a

Tabela 1. Planejamento experimental das misturas.

\begin{tabular}{ccc}
\hline Mistura & \multicolumn{2}{c}{ Componentes (proporção p/p) } \\
\hline 1 & $\mathrm{x} 1$ & $\mathrm{x} 2$ \\
\hline 2 & 1,0 & 0,0 \\
3 & 0,9 & 0,1 \\
4 & 0,8 & 0,2 \\
5 & 0,7 & 0,3 \\
\hline
\end{tabular}

x1 = gordura do leite; $\mathrm{x} 2$ = óleo de girassol. um rotaevaporador em banho de água a aproximadamente $95{ }^{\circ} \mathrm{C}$. A essa porção foi misturado $0,5 \%(\mathrm{~m} / \mathrm{m})$ de catalisador metóxido de sódio em pó (Merck, Alemanha). A reação de interesterificação foi realizada sob agitação constante, sob pressão reduzida a $70{ }^{\circ} \mathrm{C}$, em balão de três bocas imerso em banho de água, por 1 hora. Para interromper a reação foi adicionada água destilada. Para minimizar o escurecimento decorrente da reação e para absorver a umidade, foram adicionados sílica em pó (Merck, Alemanha) e sulfato de sódio anidro (Labsynth, Brasil), respectivamente. Os reagentes foram filtrados a quente utilizando-se papel de filtro (RODRIGUES; GIOIELLI, 2003).

\section{Ponto de amolecimento}

O ponto de amolecimento foi determinado pelo método do tubo capilar aberto, imerso em água sob agitação e aquecimento, de acordo com o método oficial Cc 3-25 da AOCS (1989). Os resultados expressam a média de três determinações.

\section{Consistência}

A consistência das amostras foi determinada utilizando o equipamento analisador de textura TA-XT2 (Stable Micro Systems, Inglaterra), controlado pelo programa Texture Expert versão 1.22 (Stable Micro Systems, Inglaterra). As misturas foram aquecidas em forno de micro-ondas até a temperatura de 60-70 ${ }^{\circ} \mathrm{C}$, para a fusão completa dos cristais, e acondicionadas em béqueres de $100 \mathrm{~mL}$. O condicionamento foi efetuado por 24 horas em geladeira comum $\left(5-8^{\circ} \mathrm{C}\right)$ para a recristalização da gordura e posteriormente por 24 horas em estufa em temperatura controlada. Foi utilizado cone de acrílico com ponta não truncada e ângulo de $45^{\circ}$. Os testes foram operados nas seguintes condições (D’AGOSTINI; FERRAZ; GIOIELLI, 2000): Retorno ao início; Distância $=10 \mathrm{~mm}$; Velocidade $=$ $2 \mathrm{~mm} / \mathrm{s}$; Tempo = $5 \mathrm{~s}$; Determinação da força em compressão (gf); Os resultados expressam a média de três determinações.

Para o cálculo da consistência, foi utilizada na Equação (1), proposta por Haighton (1959):

$C=K \frac{W}{p^{1,6}}$

em que:

$\mathrm{C}=$ consistência $\left(\mathrm{gf} . \mathrm{cm}^{-2}\right)$;

$\mathrm{K}$ = fator dependente do ângulo do cone;

$\mathrm{W}$ = força em compressão (gf), para tempo de 5 segundos; e

$\mathrm{p}=$ profundidade de penetração $(\mathrm{mm} / 10)$.

\section{Conteúdo de gordura sólida}

O conteúdo de gordura sólida foi analisado por ressonância magnética nuclear, utilizando aparelho Maran Ultra Bench Top $\mathrm{NMR}$, de $20 \mathrm{MHz}$, (Universal Systems, EUA) segundo normas da AOCS (1999), método Cd 16b-93. Os resultados expressam a média de duas determinações. 


\section{Microscopia sob luz polarizada}

As amostras foram fundidas a $80^{\circ} \mathrm{C}$ em forno de microondas e, utilizando-se um tubo capilar, uma gota de amostra foi colocada sobre uma lâmina de vidro. Em seguida, a gota foi coberta com uma lamínula, resultando em uma fina película de gordura. As lâminas, lamínulas e capilares foram previamente aquecidos a $80^{\circ} \mathrm{C}$.

Visando o estudo de cristalização dinâmica, as amostras foram mantidas a $80{ }^{\circ} \mathrm{C}$ durante 30 minutos para apagar a memória cristalina (MAZZANTI et al., 2004) e, em seguida, foram resfriadas a $20^{\circ} \mathrm{C}$, a $5^{\circ} \mathrm{C} /$ minuto. A esta temperatura foram capturadas as imagens em sequência para a edição dos vídeos.

As lâminas foram observadas ao microscópio de luz polarizada com auxílio de equipamento controlador de temperatura Modelo TS-4 Controller (Physitemp, EUA) acoplado a banho térmico Modelo TB-85 (Shimadzu, Japão). Foi utilizado aumento de 40 vezes no microscópio de luz polarizada Modelo BX50 (Olympus, Japão) para análise da estrutura cristalina. $\mathrm{O}$ microscópio é acoplado a uma câmera digital CoolSnap-Pro cf Color (Media Cybernetics, EUA), que transmite as imagens ao vivo para o computador utilizando o software Image Pro-Plus versão 4.5.1 (Media Cybernetics, EUA).

No processo de cristalização a $20^{\circ} \mathrm{C}$ com captura de imagens em sequência, foram tomadas imagens desde o surgimento do primeiro núcleo cristalino, em intervalos de 1 minuto. Em todos os casos, foram tomadas 100 imagens de cada processo (100 minutos). Foi determinado o diâmetro médio dos cristais, utilizando-se o software Image Pro-Plus versão 4.5.1 (Media Cybernetics, EUA) (SIMÕES; GIOIELLI, 1999, 2000; SOTERO-SOLIS; GIOIELLI, 2001; GIOIELLI; SIMÕES; RODRIGUES, 2003). Os resultados expressam a média de duas determinações.

Foram construídas curvas de cinética de cristalização das amostras a partir dos diâmetros dos cristais em função do tempo. Para traçar esta curva, foram utilizados os diâmetros dos cristais a partir de $\mathrm{t}=0$, em intervalos de 10 minutos.

Os vídeos foram elaborados com a sequência de 100 imagens obtidas em cada processo, utilizando-se o software Windows Movie Maker versão 5.1 (Microsoft, EUA).

\section{Resultados e discussão}

Apesar do conceito que o consumo de manteiga não é recomendado para pessoas com altos níveis de colesterol sanguíneo ou com alto risco de doenças cardíacas coronarianas, a gordura do leite apresenta características de qualidade interessantes para o desenvolvimento de novos produtos. Para aumentar o teor de ácidos graxos poli-insaturados e melhorar a plasticidade da manteiga, Rodrigues e Gioielli (2003) propuseram a mistura e interesterificação da gordura do leite com óleo de milho. A possibilidade de produzir alimentos nutricionalmente melhorados e com características físicoquímicas adequadas a partir da gordura do leite, estearina de palma e óleos de algodão e arroz foram avaliados por Krishna et al. (2007), utilizando métodos de modificação como o fracionamento, a mistura e a interesterificação. Cisneros et al.
(2006) avaliaram as transformações polimórficas de misturas de frações da gordura do leite de alto e baixo pontos de fusão. Foi observado que o conhecimento sobre as propriedades físicas macroscópicas da gordura do leite e suas frações é importante para muitos processos industriais.

A Tabela 2 apresenta os resultados de conteúdo de gordura sólida, consistência e ponto de amolecimento das misturas de gordura do leite e óleo de girassol, antes e após a interesterificação química. Os valores de conteúdo de gordura sólida e consistência são apresentados somente a $20^{\circ} \mathrm{C}$, uma vez que o acompanhamento da cristalização por microscopia sob luz polarizada foi efetuado a esta temperatura.

A Figura 1 é referente aos valores de conteúdo de gordura sólida, consistência e ponto de amolecimento em relação à proporção de óleo de girassol presente nas misturas com gordura do leite.

A Figura 1(A) mostra a variação no conteúdo de gordura sólida com a adição de óleo de girassol nas misturas com gordura do leite, antes e após a interesterificação. O conteúdo de gordura sólida das misturas e dos lipídios estruturados diminuiu com a adição de óleo de girassol, em função do efeito de diluição da gordura do leite com o óleo líquido. Os lipídios estruturados apresentaram, de modo geral, conteúdo de gordura sólida superior ao verificado nas misturas, como decorrência do rearranjo ao acaso entre os triacilgliceróis presentes.

A Figura 1(B) indica que a adição de óleo de girassol também provocou diminuição na consistência da gordura do leite. O óleo de girassol é líquido nas temperaturas analisadas e ocasionou a diluição da rede cristalina da gordura do leite, diminuindo a interação cristalina e, por consequência, a consistência. Assim como observado para o conteúdo de gordura sólida, de modo geral a consistência dos lipídios estruturados foi superior à apresentada pelas misturas.

Tabela 2. Conteúdo de gordura sólida, consistência e ponto de amolecimento $^{1}$ das misturas de gordura do leite e óleo de girassol, antes e após a interesterificação química.

\begin{tabular}{ccccc}
\hline $\begin{array}{c}\text { Amostras } \\
\left(\mathrm{n}^{\circ}\right)\end{array}$ & $\begin{array}{c}\text { Conteúdo de } \\
\text { gordura sólida }(\%) \\
\text { a } 20{ }^{\circ} \mathrm{C}\end{array}$ & $\begin{array}{c}\text { Consistência } \\
\left(\text { gf.cm }^{-2}\right) \\
\text { a } 20{ }^{\circ} \mathrm{C}\end{array}$ & $\begin{array}{c}\text { Ponto de } \\
\text { amolecimento } \\
\left({ }^{\circ} \mathrm{C}\right)\end{array}$ \\
\hline 1 & M & $19,0 \pm 0,0$ & $922,2 \pm 14,2$ & $33,1 \pm 0,2$ \\
& IQ & $21,3 \pm 0,6$ & $3717,1 \pm 236,4$ & $37,1 \pm 0,2$ \\
2 & M & $16,1 \pm 0,4$ & $576,9 \pm 10,1$ & $31,8 \pm 0,2$ \\
& IQ & $21,2 \pm 0,6$ & $1448,9 \pm 111,8$ & $34,1 \pm 0,1$ \\
3 & M & $12,8 \pm 0,6$ & $377,8 \pm 10,6$ & $31,3 \pm 0,2$ \\
& IQ & $16,1 \pm 1,0$ & $964,5 \pm 9,2$ & $33,5 \pm 0,2$ \\
4 & M & $11,1 \pm 1,1$ & $207,5 \pm 4,9$ & $31,3 \pm 0,4$ \\
& IQ & $11,6 \pm 0,3$ & $509,1 \pm 24,3$ & $32,0 \pm 0,1$ \\
5 & M & $8,3 \pm 0,4$ & 0 & $30,7 \pm 0,2$ \\
& IQ & $8,1 \pm 1,1$ & $176,5 \pm 2,7$ & $31,0 \pm 0,2$ \\
\hline
\end{tabular}

${ }^{1}$ média \pm desvio-padrão.

$\mathrm{M}=$ mistura; $\mathrm{IQ}$ = interesterificação química 
O ponto de amolecimento das misturas também decresceu com o aumento na proporção de óleo de girassol, como pode ser observado na Figura 1(C), embora em menor proporção. Isto ocorre porque o ponto de amolecimento depende da passagem para o estado líquido dos triacilgliceróis de maior ponto de fusão, que ainda estão presentes em maior proporção quando o teor de gordura do leite nas misturas é maior que 60\%. Assim como observado para o conteúdo de gordura sólida e para a consistência, os lipídios estruturados apresentaram maiores pontos de amolecimento que as misturas correspondentes. $\mathrm{O}$ aumento no ponto de amolecimento de misturas contendo gordura do leite interesterificadas quimicamente também foi observado por Rousseau e Marangoni (1999). Segundo estes autores, foi observada uma relação diretamente proporcional entre o teor de triacilgliceróis trissaturados e o ponto de amolecimento.

$\mathrm{Na}$ análise sob luz polarizada, a fase sólida anisotrópica da rede cristalina refrata a luz de forma diferente que a fase líquida isotrópica. A fase líquida aparece nas imagens em preto e a fase sólida em branco ou em tons de cinza (NARINE; MARANGONI, 1999c).

A Figura 2 apresenta imagens digitalizadas das amostras cristalizadas à temperatura de $20^{\circ} \mathrm{C}$, após 100 minutos do início da tomada das imagens.

A Figura 3 é referente à variação no diâmetro dos cristais presentes nas gorduras antes e após a interesterificação. Esses dados indicam o comportamento de cristalização de cada amostra ao longo do tempo de experimento sob temperatura constante de $20^{\circ} \mathrm{C}$.

Conforme pode se observado na Figura 2, as amostras compostas por misturas de gordura de leite e óleo de girassol apresentaram diferenças na forma cristalina, no tamanho e no número dos cristais em função da diluição com o óleo de girassol e da interesterificação. Com o aumento da proporção de óleo de girassol pôde ser observado que a diluição da gordura do leite com o óleo líquido ocasionou a diminuição do número dos cristais, com correspondente aumento de diâmetro deles. Este fenômeno é resultado da diminuição das barreiras físicas que impedem o crescimento dos cristais, principalmente pela presença de maior quantidade de matriz líquida composta pelo óleo de girassol. A interesterificação química alterou a característica da rede cristalina quando comparada com os padrões da cristalização antes da reação. Os esferulitos observados nas misturas foram do tipo A, caracterizados por cristais com núcleo compacto, cercado de agulhas longas e finas distribuídas radialmente. Por outro lado, os esferulitos observados nos lipídios estruturados foram do tipo $\mathrm{B}$, caracterizados por pequenos núcleos cercados de cristais orientados aleatoriamente (BERGER; JEWELL; POLLITT, 1979).

Após a interesterificação química ocorreu alteração nas propriedades reológicas das amostras, caracterizada pelo efeito de diluição das misturas pela incorporação dos ácidos graxos insaturados do óleo de girassol pelos triacilgliceróis da gordura do leite (ROUSSEAU; HILL; MARANGONI, 1996b).

O óleo de girassol não cristaliza em temperaturas acima de $-10{ }^{\circ} \mathrm{C}$ e portanto não está diretamente envolvido com a cristalização da gordura do leite. Devido ao fato do óleo de girassol apresentar cerca de $91 \%$ de ácidos graxos com 18 átomos de carbono, ele pode ter hidrofobicidade ligeiramente maior que a gordura do leite. As diferenças no tamanho médio das cadeias de ácidos graxos da gordura do leite e do óleo de girassol possivelmente podem explicar as estruturas observadas. Durante a cristalização, os triacilgliceróis do óleo de girassol
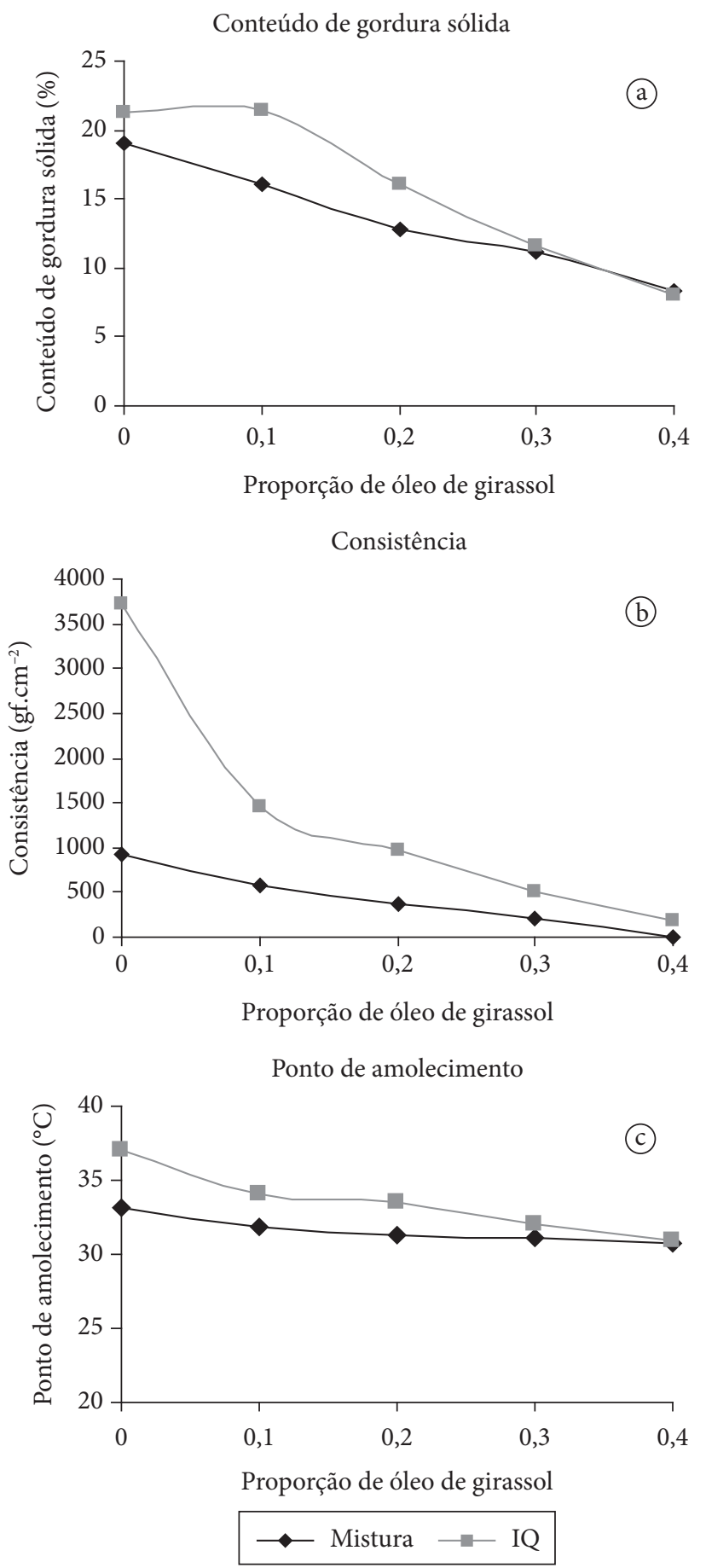

Figura 1. Relação entre propriedades físicas e a proporção de óleo de girassol na mistura com a gordura do leite, antes e após a interesterificação. 


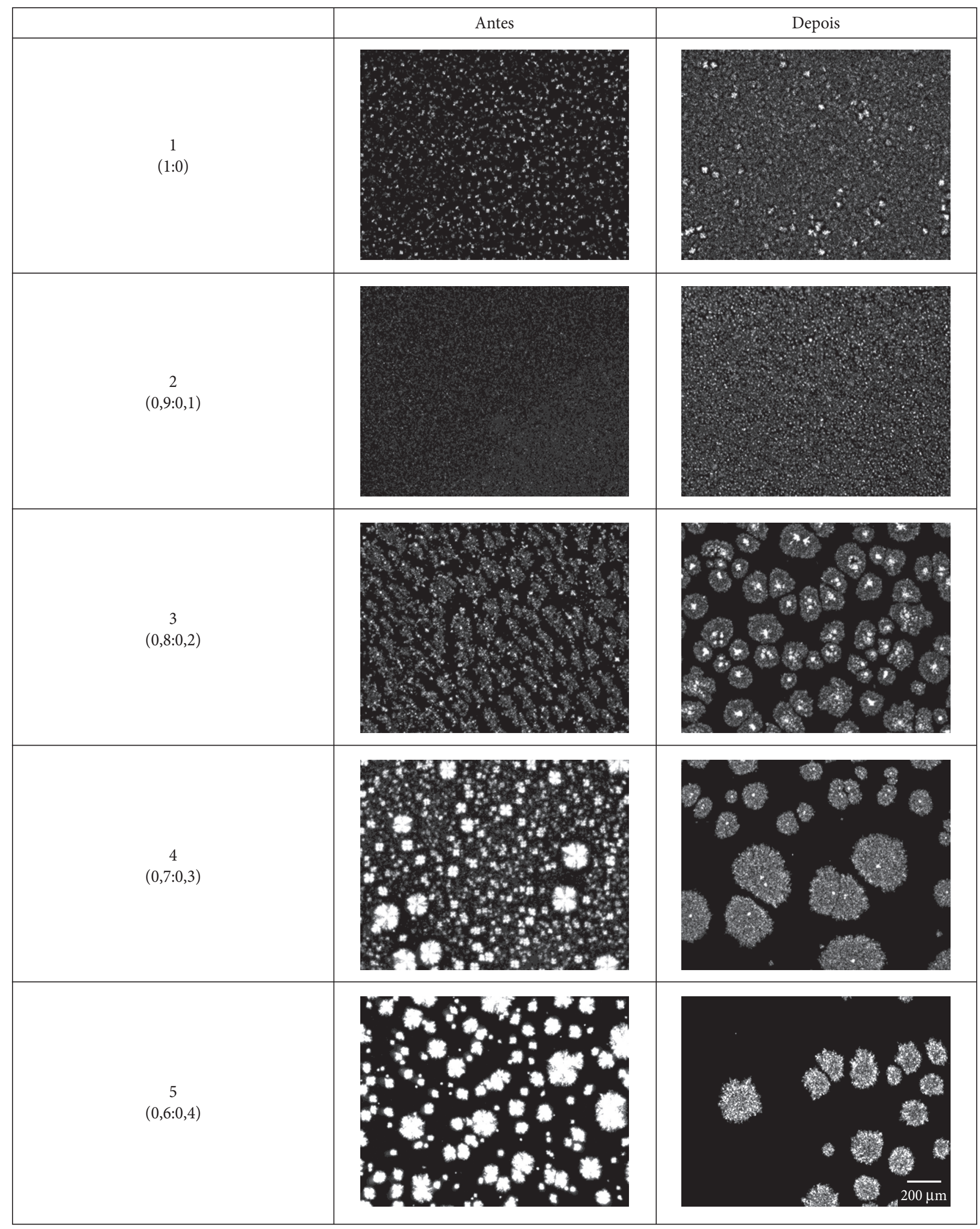

Figura 2. Estrutura cristalina das amostras observadas na última imagem dos vídeos de cristalização a $20^{\circ} \mathrm{C}$ das misturas de gordura do leite com óleo de girassol, com aumento de 40x. A barra representa $200 \mu \mathrm{m}$. 
teriam dificuldade em se dispersar entre as moléculas de gordura do leite que estão em fase de cristalização. Portanto, a formação de esferulitos seria mais provável (ROUSSEAU; HILL; MARANGONI, 1996a).

A Figura 3(A) indica o aumento do diâmetro dos cristais em função do tempo, especialmente para as misturas contendo maior quantidade de óleo de girassol, uma vez que nestes casos a cristalização é mais lenta. O diâmetro dos cristais atingiu valores da ordem de $250 \mu \mathrm{m}$ para as misturas de gordura do leite com óleo de girassol. Após a interesterificação química, conforme apresentado na Figura 3(B), os diâmetros aumentaram, atingindo valores da ordem de $400 \mu \mathrm{m}$ para as misturas de gordura do leite com óleo de girassol.

Não foi possível obter correlação entre as propriedades de consistência, conteúdo de gordura sólida e ponto de amolecimento com os resultados de diâmetro dos cristais. Isto
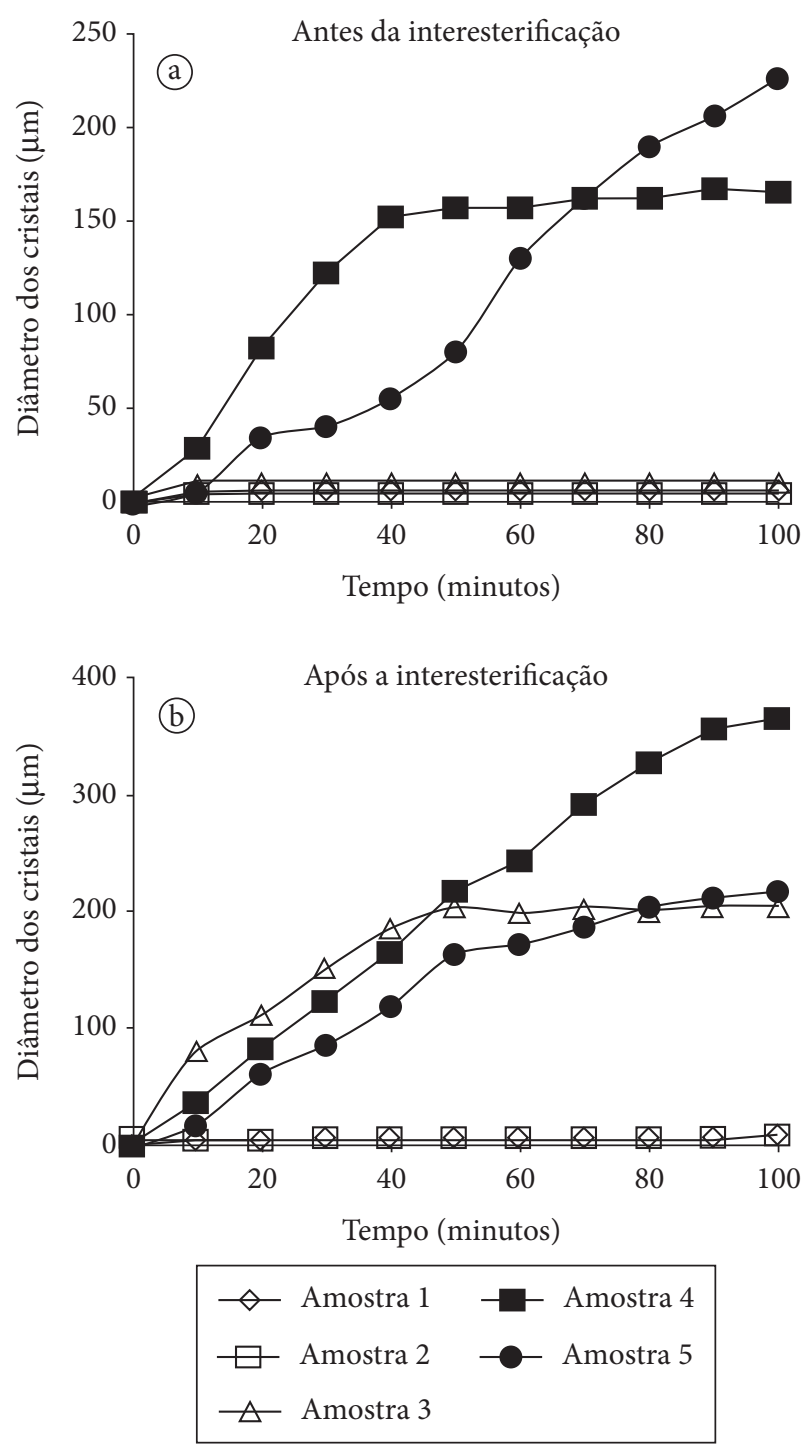

Figura 3. Diâmetro dos cristais em função do tempo de cristalização a $20^{\circ} \mathrm{C}$ das misturas de gordura do leite e óleo de girassol. mostra que a cristalização é um fenômeno complexo e que outros fatores devem estar envolvidos na determinação das propriedades físicas dos óleos e gorduras.

A utilização de vídeos ou sequências de imagens para o estudo da cinética de cristalização de lipídios (BATTE; MARANGONI, 2005; DIBILDOX-ALVARADO et al., 2004; LITWINENKO; SINGH; MARANGONI, 2004) consiste em metodologia que ainda não está totalmente estabelecida e, portanto, são necessárias algumas observações a respeito do comportamento das amostras durante os experimentos.

Todos os tratamentos térmicos empregados às lâminas contendo as amostras apresentavam uma fase inicial comum, que consistia de 30 minutos de isoterma a $80^{\circ} \mathrm{C}$ para eliminação da memória cristalina, seguida de resfriamento rápido a $5{ }^{\circ} \mathrm{C} /$ minuto de 40 para $20^{\circ} \mathrm{C}$. As amostras não apresentavam nenhum sinal de cristalização, o que confirmava que elas haviam sido completamente fundidas nesta temperatura. De acordo com Marangoni (2005), para a maioria dos óleos e gorduras comestíveis, 30 minutos a $80{ }^{\circ} \mathrm{C}$ são suficientes para apagar a memória cristalina de gorduras. Porém, a experiência prática mostrou que a agitação vigorosa das amostras após fusão completa é um procedimento complementar que garante a total desorganização das moléculas que se apresentavam organizadas formando cristais. Desse modo, a preparação de lâminas de gordura para microscopia deve ser feita utilizando-se todo o material necessário (lâminas, lamínulas e capilares) previamente aquecido a temperaturas o mais próximo possível de $80{ }^{\circ} \mathrm{C}$ (NARINE; MARANGONI, 1999a; ROUSSEAU; MARANGONI; JEFFREY, 1998). Assim, é possível evitar a cristalização das amostras no momento do contato com o material de preparação, garantindo que sua memória estará completamente apagada no início do processo de cristalização.

A cristalização da gordura do leite é mais complicada do que a maioria das outras gorduras devido à sua complexidade molecular, à formação de cristais mistos e às diferentes modificações polimórficas (METIN; HARTEL, 1998). Além disso, a gordura do leite não é um produto refinado e contém, naturalmente, fosfolipídios e esteróis que podem influenciar sua estrutura cristalina (HUI, 1996).

Os vídeos correspondentes a cada sequência estão disponíveis para visualização na Internet, http://www.fcf. usp.br/Departamentos/FBT/HP_Professores/Gioielli/Videos/ Index.asp.

Nessas imagens, podem-se observar as diferentes formas cristalinas obtidas com as amostras submetidas às mesmas condições de tratamento térmico.

Como exemplos, podem ser citados os vídeos correspondentes às amostras 4 e 5 , compostas por $70 \%$ de gordura do leite e $30 \%$ de óleo de girassol e $60 \%$ de gordura do leite e $40 \%$ de óleo de girassol, respectivamente. O conteúdo de gordura sólida e o ponto de amolecimento destas amostras, antes e após a interesterificação, foram muito próximos. No entanto, após a interesterificação a consistência apresentou valor muito mais elevado (Tabela 2). Os vídeos correspondentes claramente demonstram formas distintas de cristalização, que podem justificar a grande diferença na consistência em decorrência 
do rearranjo ao acaso. Contudo, deve ser lembrado que, para a determinação do conteúdo de gordura sólida e da consistência, as amostras passam por condicionamento prévio em temperaturas baixas, enquanto que nos vídeos são demonstrados os processos de cristalização passando diretamente do estado líquido para o sólido a $20^{\circ} \mathrm{C}$. O conteúdo de gordura sólida é considerado um fator principal que influencia as propriedades reológicas de sistemas lipídicos semissólidos. Altos valores de conteúdo de gordura sólida geralmente levam a produtos duros. Contudo, sistemas com o mesmo conteúdo de gordura sólida podem ter propriedades reológicas muito diferentes, indicando que a microestrutura do sistema também tem importante impacto nas propriedades reológicas, como dureza e espalhabilidade (LIANG et al., 2006), o que foi evidenciado nas amostras 4 e 5, como relatado acima.

Além da complexa composição em ácidos graxos e triacilgliceróis, a cristalização da gordura do leite a partir do estado líquido pode ser afetada por vários parâmetros experimentais, incluindo a velocidade de agitação, a escala de operação, a temperatura final e a velocidade de resfriamento (GRALL; HARTEL, 1992).

Outro fenômeno que pôde ser observado durante os experimentos foi a cristalização das amostras apenas após o término das rampas de temperatura, ou seja, as amostras não iniciaram seu processo de cristalização enquanto estavam sendo resfriadas. Os primeiros cristais surgiam apenas depois que as amostras haviam atingido $20^{\circ} \mathrm{C}$.

Lopez et al. (2005) realizaram o resfriamento da gordura do leite a $3{ }^{\circ} \mathrm{C} /$ minuto e observaram, por difração de raios $\mathrm{X}$, que, na faixa de temperatura $60 \geq \mathrm{T} \geq 19^{\circ} \mathrm{C}$, a gordura do leite se encontrava na forma líquida. Em outro estudo, Batte e Marangoni (2005) resfriaram lâminas de gordura do leite a partir de 80 até $21^{\circ} \mathrm{C}$ à velocidade de $5^{\circ} \mathrm{C} /$ minuto e também não puderam iniciar a tomada dos resultados antes de estar finalizado o processo de resfriamento, ou seja, antes da amostra atingir $21^{\circ} \mathrm{C}$.

Uma possível justificativa para o fenômeno em questão está no fato de que a gordura do leite possui uma das composições em ácidos graxos mais complexas dentre as conhecidas até o momento (HERRERA; GATTI; HARTEL, 1999). Devido à grande diversidade de ácidos graxos presentes na gordura do leite, o crescimento dos cristais é dificultado pela competição de triacilgliceróis similares, porém incompatíveis, que se incorporam em uma rede que não se empacota adequadamente ou voltam a se difundir na fase líquida. Em ambos os casos, o crescimento de cristais já existentes é retardado devido à presença de muitos triacilgliceróis diferentes (GRALL; HARTEL, 1992).

A gordura do leite pode cristalizar em 4 formas distintas, dependendo das condições de resfriamento: sub- $\alpha, \alpha, \beta$-prima e $\beta$, sendo que a transição sub- $\alpha \rightarrow \alpha$ é reversível e as transições $\alpha \rightarrow \beta$-prima $\rightarrow \beta$ (em ordem crescente de estabilidade) são irreversíveis. De qualquer maneira, independentemente da velocidade de resfriamento, várias organizações cristalinas diferentes são encontradas simultaneamente na gordura do leite. As formas a e $\beta$-prima são as mais comumente encontradas e a forma $\beta$ já foi encontrada em traços, principalmente em processos muito lentos de resfriamento e na presença de grande fração líquida, características que favorecem a formação de cristais mais estáveis (LOPEZ et al., 2005).

As principais forças que levam à formação de redes cristalinas de gorduras são as forças atrativas de Van der Waals (ROUSSEAU; HILL; MARANGONI, 1996b). A migração dos cristais observada em alguns vídeos pode ser devida a essas forças. Uma segunda possível explicação para este fato é a diferença de densidade e, consequentemente, de volume, entre lipídios no estado sólido e no estado líquido. Quando uma gordura passa do estado líquido para o sólido, ela se torna mais densa e seu volume, portanto, diminui (GERMAN; SIMONEAU, 1998). Essas mudanças de volume decorrentes das mudanças de fase poderiam causar movimentação da fração líquida existente, que acaba por arrastar pequenos cristais durante sua movimentação.

Portanto, a técnica de captura de imagens em sequência durante a cristalização de gorduras permite acompanhar as transformações dinâmicas que estão ocorrendo no processo, o que não pode ser obtido quando se realiza o estudo por microscopia sob luz polarizada após um tempo pré-determinado de cristalização.

\section{Conclusões}

Os resultados observados demonstraram o efeito de diluição da gordura do leite com a adição de óleo de girassol, visto que, na temperatura de $20{ }^{\circ} \mathrm{C}$, houve aumento na matriz líquida o que permitiu que os cristais crescessem em diâmetro, porém diminuíssem em número, pois ficaram mais dispersos.

A interesterificação intensificou os efeitos de diluição pela redistribuição e incorporação de ácidos graxos insaturados nas moléculas dos triacilgliceróis da gordura do leite. Estes resultados influenciaram diretamente nas propriedades reológicas das misturas de lipídios.

Os esferulitos observados nas misturas foram do tipo A, caracterizados por cristais com núcleo compacto, cercado de agulhas longas e finas distribuídas radialmente. Por outro lado, os esferulitos observados nos lipídios estruturados foram do tipo B, caracterizados por pequenos núcleos cercados de cristais orientados aleatoriamente.

Foram realizados vídeos constituídos por sequências de imagens de cristalização de cada amostra. Este método permitiu a visualização dos fenômenos físicos envolvidos na cristalização das misturas de gorduras e seus correspondentes lipídios estruturados. A técnica de captura de imagens em sequência durante a cristalização de gorduras permitiu acompanhar as transformações dinâmicas que ocorreram no processo, o que não pode ser obtido quando se realiza o estudo por microscopia sob luz polarizada após um tempo pré-determinado de cristalização. 


\section{Agradecimentos}

À FAPESP, CAPES e CNPq pelo apoio financeiro e bolsas concedidas aos autores.

\section{Referências bibliográficas}

AMERICAN OIL CHEMISTS' SOCIETY - AOCS. Official methods and recommended practices of the AOCS. Champaign, 1989.

AMERICAN OIL CHEMISTS' SOCIETY - AOCS. Official methods and recommended practices of the AOCS. Champaign, 1999.

BATTE, H. D.; MARANGONI, A. G. Fractal growth of milk fat crystals is unaffected by microstructural confinement. Crystal Growth and Design, v. 5, n. 5, p. 1703-1705, 2005.

BERGER, K. G.; JEWELL, G. G.; POLLITT, R. J. M. Oils and fats. In: VAUGHAN, J. G. Food microscopy. London: Academic Press, 1979. p. 445-497.

CAMPOS, R.; NARINE, S. S.; MARANGONI, A. G. Effect of cooling rate on structure and mechanical properties of milk fat and lard. Food Research International, v. 35, n. 10, p. 971-981, 2002.

CISNEROS, A. et al. Polymorphic transformation in mixtures of highand low-melting fractions of milk fat. Journal Agricultural Food Chemistry, v. 54, n. 16, p. 6030-6033, 2006.

D’AGOSTINI, D.; FERRAZ, R. C.; GIOIELLI, L. A. Consistência de misturas binárias e ternárias de gorduras de palma, palmiste e triacilgliceróis de cadeia média. Revista Brasileira de Ciências Farmacêuticas, v. 36, n. 1, p. 147-155, 2000.

DÍAZ GAMBOA, O. W.; GIOIELLI, L. A. Comportamento de cristalização de lipídios estruturados obtidos a partir de gordura de palmiste e óleo de peixe. Química Nova, v. 29, n. 4, p. 646-653, 2006.

DIBILDOX-ALVARADO, E. et al. Effects of crystalline microstructure on oil migration in a semisolid fat matrix. Crystal Growth and Design, v. 4, n. 4, p. 731-736, 2004.

GERMAN, J. B.; SIMONEAU, C. Phase transitions of edible fats and triglycerides: theory and applications. In: RAO, M. A.; HARTEL, R. W. Phase/State transitions in foods. New York: Marcel Dekker, 1998. p. 187-216.

GHOTRA, B. S.; DYAL, S. D.; NARINE, S. S. Lipid shortenings: a review. Food Research International, v. 35, n. 10, p. 1015-1048, 2002.

GIOIELLI, L. A.; SIMÕES, I. S.; RODRIGUES, J. N. Crystal morphology and interactions of binary and ternary mixtures of hydrogenated fats. Journal Food Engineering, v. 57, n. 4, p. 347-355, 2003.

GRALL, D. S.; HARTEL, R. W. Kinetics of butterfat crystallization. Journal American Oil Chemists' Society, v. 69, n. 8, p. 741-747, 1992.

HAIGHTON, A. J. The measurement of the hardness of margarine and fats with cone penetrometers. Journal American Oil Chemists' Society, v. 36, n. 8, p. 345-348, 1959.

HERRERA, M. L. et al. Isothermal crystallzation of hydrogenated sunflower oil: 1. Nucleation. Journal American Oil Chemists' Society, v. 75, n. 10, p. 1273-1280, 1998.

HERRERA, M. L.; GATTI, M. L.; HARTEL, R. W. A kinetic analysis of crystallization of a milkfat model system. Food Research International, v. 32, n. 4, p. 289-298, 1999b.

HIMAWAN, C.; STAROV, V. M.; STAPLEY, A. G. F. Thermodynamic and kinetic aspects of fat crystallization. Advances Colloid Interface Science, v. 122, n. 1-3, p. 3-33, 2006.

HUI, Y. H. Bailey's industrial oil and fat products. 5 ed. New York: Wiley-Interscience, 1996. (v. 2 e v. 3)
KRISHNA, B. et al. Plastic fats and margarines through fractionation, blending and interesterification of milk fat. European Journal Lipid Science Technology, v. 109, n. 1, p. 32-37, 2007.

LIANG, B. et al. Approches to quantification of microstructure for model lipid systems.Journal American Oil Chemists' Society, v. 83, n. 5, p. 389-399, 2006.

LITWINENKO, J. W.; SINGH, A. P.; MARANGONI, A. G. Effects of glycerol and Tween 60 on the crystallization behavior, mechanical properties, and microstructure of a plastic fat. Crystal Growth and Design v. 4, n. 1, p. 161-168, 2004.

LOPEZ, C. et al. Thermal and structural behavior of anhydrous milk fat. 3. Influence of cooling rate. Journal Dairy Science, v. 88, n. 2, p. 511-526, 2005.

MARANGONI, A. G. Fat crystal networks. New York: Marcel Dekker, 2005. $854 \mathrm{p}$.

MARANGONI, A. G.; ROUSSEAU, D. Chemical and enzymatic modification of butterfat and butterfat-canola oil blends. Food Research International, v. 31, n. 8, p. 595-599, 1998a.

MAZZANTI, G. et al. Effect of minor components and temperature profiles on polymorphism in milk fat. Crystal Growth and Design, v. 4, n. 6, p. 1303-1309, 2004.

METIN, S.; HARTEL, R. W. Thermal analysis of isothermal crystallization kinetics in blends of cocoa butter with milk fat or milk fat fractions. Journal American Oil Chemists' Society, v. 75, n. 11, p. 1617-1624, 1998.

NARINE, S. S.; MARANGONI, A. G. Microscopic and rheological studies of fat crystal networks. Journal Crystal Growth, v. 198-199, n. 2, p. 1315-1319, 1999c.

NARINE, S. S.; MARANGONI, A. G. Relating structure of fat crystal networks to mechanical properties: a review. Food Research International, v. 32, n. 4, p. 227-248, 1999d.

NARINE, S. S.; MARANGONI, A. G. Factors affecting the texture of plastic fats. INFORM, v. 10, n. 6, p. 565-570, 1999a.

PISKA, I. et al. Properties and crystallization of fat blends. Journal Food Engineering, v. 77, n. 3, p. 433-438, 2006.

RODRIGUES, J. N. Reestruturação da gordura do leite por mistura e interesterificação com óleo de milho. 2002. 119 p. Dissertação (Mestrado) - Universidade de São Paulo, São Paulo.

RODRIGUES, J. N.; ANTON, C.; GIOIELLI, L. A. Cristalização de lipídios estruturados obtidos a partir de gordura do leite e óleo de milho. Revista Brasileira Ciências Farmacêuticas, v. 39, n. 1, p. 93-103, 2003a.

RODRIGUES, J. N.; GIOIELLI, L. A. Chemical interesterification of milkfat and milkfat-corn oil blends. Food Research International, v. 36, n. 2, p. 149-159, 2003.

RODRIGUES, J. N.; GIOIELLI, L. A.; ANTON, C. Propriedades físicas de lipídios estruturados obtidos de misturas de gordura do leite e óleo de milho. Ciência Tecnologia Alimentos, v. 23, n. 2, p. 226-233, 2003b.

RODRIGUES, J. N. et al. Caracterização físico-química de creme vegetal enriquecido com ésteres de fitosteróis. Revista Brasileira Ciências Farmacêuticas, v. 40, n. 4, p. 505-520, 2004.

ROUSSEAU, D. et al. Restructuring butterfat through blending and chemical interesterification: 1. Melting behavior and triacylglycerol modifications. Journal American Oil Chemists' Society, v. 73, n. 8, p. 963-972, 1996a.

ROUSSEAU, D.; HILL, A. R.; MARANGONI, A. G. Restructuring butterfat through blending and chemical interesterification: 
2. Microstructure and polymorphism. Journal American Oil Chemists' Society, v. 73, n. 8, p. 973-981, 1996 b.

ROUSSEAU, D.; HILL, A. R.; MARANGONI, A. G. Restructuring butterfat through blending and chemical interesterification: 3 . Rheology. Journal American Oil Chemists' Society, v. 73, n. 8, p. 983-989, 1996c.

ROUSSEAU, D.; MARANGONI, A. G. The effects of interesterification on physical and sensory attributes of butterfat and butterfat-canola oil spreads. Food Research International, v. 31, n. 5, p. 381-388, 1999.

ROUSSEAU, D.; MARANGONI, A. G.; JEFFREY, K. R. The influence of chemical interesterification on physicochemical properties of complex fat systems: 2. Morphology and polymorphism. Journal American Oil Chemists' Society, v. 75, n. 12, p.1833-1839, 1998.

SATO, K. Crystallization behaviour of fats and lipids - a review. Chemical Engineering Science, v. 56, n. 7, p. 2255-2265, 2001.

SILVA, R. C.; ESCOBEDO, J. P.; GIOIELLI, L. A. Comportamento de cristalização de lipídios estruturados por interesterificação química de banha e óleo de soja. Química Nova, v. 31, n. 2, p. 330-335, 2008.

SIMÕES, I. S.; GIOIELLI, L. A. Microscopia da cristalização parcial de misturas de gorduras hidrogenadas e óleo de soja. Revista Brasileira Ciências Farmacêuticas, v. 35, n. 2, p. 259-266, 1999.
SIMÕES, I. S.; GIOIELLI, L. A. Crystal morphology of binary and ternary mixtures of hydrogenated fats and soybean oil. Brazilian Archives Biology Technology, v. 43, n. 2, p. 241-248, 2000.

SOTERO-SOLIS, V. E.; GIOIELLI, L. A. Estrutura cristalina de las grasas hidrogenadas del aceite de castaña de Brasil (Bertholletia excelsa). Alimentaria, v. 38, n. 322, p. 131-137, 2001.

TANG, D.; MARANGONI, A. G. Quantitative study on the microstructure of colloidal fat crystal networks and fractal dimensions. Advances Colloid Interface Science, v. 128-130, n. 1, p. 257-265, 2006.

TIMMS, R. E. Crystallization of fats. In: HAMILTON, R. J. Developments in oils and fats. London: Chapman \& Hall, 1995. p. 204-223.

TORO-VAZQUEZ, J. F. et al. Crystallization kinetics of palm stearin in blends with sesame seed oil. Journal American Oil Chemists' Society, v. 77, n. 3, p. 297-310, 2000.

WRIGHT, A. J. et al. The effect of minor components on milk fat crystallization. Journal American Oil Chemists' Society, v. 77, n. 5, p. 463-475, 2000.

WRIGHT, A. J. et al. Rheological properties of milkfat and butter. Journal Food Science, v. 66, n. 8, p. 1056-1071, 2001. 